\title{
Elastic properties of small-world spring networks
}

\author{
A. Ramezanpoul* and S. M. Vaez Allaet \\ Institute for Advanced Studies in Basic Sciences, Zanjan 45195-1159, Iran
}

(Dated: November 19, 2018)

\begin{abstract}
We construct small-world spring networks based on a one dimensional chain and study its static and quasistatic behavior with respect to external forces. Regular bonds and shortcuts are assigned linear springs of constant $k$ and $k^{\prime}$, respectively. In our models, shortcuts can only stand extensions less than $\delta_{c}$ beyond which they are removed from the network. First we consider the simple cases of a hierarchical small-world network and a complete network. In the main part of this paper we study random small-world networks (RSWN) in which each pair of nodes is connected by a shortcut with probability $p$. We obtain a scaling relation for the effective stiffness of RSWN when $k=k^{\prime}$. In this case the extension distribution of shortcuts is scale free with the exponent -2 . There is a strong positive correlation between the extension of shortcuts and their betweenness. We find that the chemical end-to-end distance (CEED) could change either abruptly or continuously with respect to the external force. In the former case, the critical force is determined by the average number of shortcuts emanating from a node. In the latter case, the distribution of changes in CEED obeys power laws of the exponent $-\alpha$ with $\alpha \leq 3 / 2$.

PACS numbers: 89.75.Fb, 05.40.-a
\end{abstract}

*Electronic address: ramezanpour@iasbs.ac.ir

$\dagger$ Electronic address: smvaez@iasbs.ac.ir 


\section{INTRODUCTION}

Recent studies indicate that real networks have a complex structure and function

11, 2, 3. 4 4, 5]. The small-world network (SWN) introduced by Watts and Strogatz [3] captures some basic ingredients of real networks. There are lots of studies dealing with static properties of SWNs, see 2] and references therein. However, we still only know a little bit about dynamical features of small-world networks [6, 7, , 8, ㅁ, 10, 11]. To best of our knowledge there is no study on the elastic properties of SWNs. Certainly the most physical application of small-world networks is in the context of macromolecules and polymers 12, 13, 14, 15, 16]. The interaction pattern of a polymer can be represented by a SWN. In this way, the monomers are mapped into nodes of a network and the interaction between two spatially close monomers is shown by a bond between the corresponding nodes. Note that here there are two kinds of bonds: regular and long range. The long range bonds that are usually weaker than the regular ones, could stand much smaller stresses and would be broken more easily. If we model the interactions with linear springs, we will obtain a small-world spring network. Of course, we should take more care on modeling linear polymers with SWNs [17]. Nevertheless, we expect that the study of small-world networks (as a toy model of polymers when thermal activities are absent) provides insights about more complicated behaviors of real polymers.

Suppose that we have a chain of elastic fibers and add some other fibers to randomly selected pairs of nodes. Then it is interesting to know , for example, the effective stiffness of this object and some other quantities which are of interest in fiber-bundle models [18, 19].

In this paper we are going to study the behavior of small-world spring networks when external force $F$ is exerted on the end nodes. The network response contains some information about its internal structure. For a given $F$, we obtain the network stiffness and the extension distribution of long range bonds (shortcuts). Then we increase $F$ quasistatically and define a cutoff length for shortcuts beyond which we remove them from the network. During this quasistatic process the chemical end-to-end distance (CEED), defined as the number of bonds in the shortest path connecting node 1 to $N$, could have nontrivial behavior with $F$. Moreover by increasing $F$ one encounters a number of avalanches in which one has a change in the number of shortcuts and also in CEED. Certainly, distinct structures could lead to different behaviors, for instance in the distribution of changes in 
CEED. These differences provide us a useful measure to classify various networks.

Here we will show that the extension distribution is scale free in the small-world regime in contrast to the three-modal distribution of complete-network regime. We obtain a positive

correlation between the betweenness [2] of a shortcut and its extension. There is also a threshold value $F_{c}$ which leads to an abrupt change in CEED. The scaling of $F_{c}$ with the size of the network depends strongly on the network structure. Depending on the elastic properties of springs we could also have a continuous change in CEED.

The structure of this paper is as follows. After giving some general definitions, we introduce and study a hierarchical small-world network in section III. In section IV we study complete networks as another simple case. In section $\nabla$ we present the results of our numerical simulations and scaling arguments for random small-world networks . Finally we give the conclusion remarks.

\section{GENERAL DEFINITIONS}

We take a one dimensional chain with $N$ nodes, numbered 1 to $N$. This chain has $N-1$ regular bonds, with spring constant $k$. The network structure is completed by adding some shortcuts of spring constant $k^{\prime}$. In the following we will consider the case $0 \leq k^{\prime} \leq k$ which is more reasonable in physical models. The effective elastic constant of the network is denoted by $K$. We exert force $F$ on the end nodes of a chain and obtain the extension distribution of shortcuts, $P\left(\Delta_{x}\right)$, where $k^{\prime} \Delta_{x}$ is the force acting on a shortcut. On increasing $F$ quasistatically, we define a cutoff length $\delta_{c}$ for shortcuts. It means that a shortcut is teared if its extension exceeds $\delta_{c}$. Here we will take $\delta_{c}=1$ (the specific value of $\delta_{c}$ does not affect the qualitative behavior of our results). For a given network, we may have a number of avalanches in some particular forces. An avalanche starts by the tearing of a shortcut and ends when all the shortcuts have an extension smaller than $\delta_{c}$. By increasing $F$, a number of avalanches may occur which we label by index $a$. In each avalanche, one can measure, for example, the force value $F_{a}$, the chemical end-to-end distance $R_{a}$ and the effective spring constant of the network $K_{a}$. The CEED, $R$, is defined as the number of bonds in the shortest path connecting node 1 to $N$. Just after the $a$-th avalanche, the physical end-toend distance, $X_{a}$, is given by $F_{a} / K_{a}$. We also define the change in a quantity such as $R$ by 


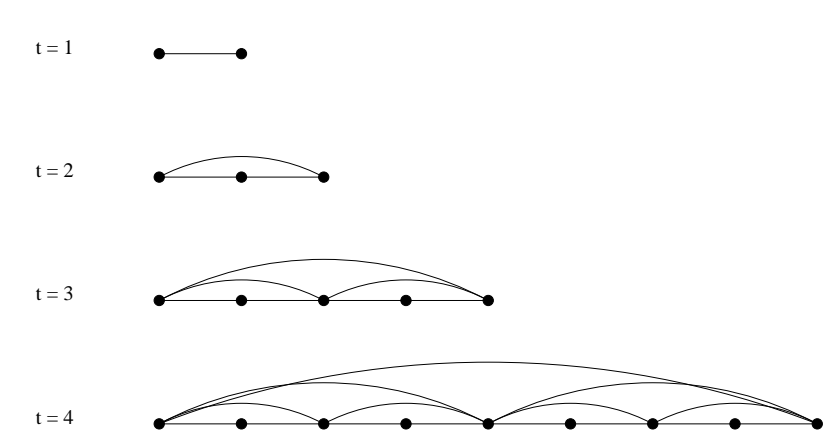

FIG. 1: Constructing a hierarchical small-world network.

$\Delta R_{a}:=R_{a+1}-R_{a}$.

\section{A HIERARCHICAL SMALL-WORLD NETWORK}

Let us first study a simple hierarchical small-world network where its behavior can be treated exactly. The construction of the network is depicted in Fig. 11. In the first step, $t=1$, we consider two nodes connected with a regular bond. In the next step, $t=2$, we make a copy of the previous step and merge them. We also add an additional shortcut between the first and last nodes of the new network. If we repeat this procedure for $t$ steps we obtain a hierarchical SWN of size $N(t)=2^{t-1}+1$ with $M(t)=2^{t-1}-1$ shortcuts. One can obtain the following relation for the effective stiffness of the network

$$
K(t)=k^{\prime}+\frac{K(t-1)}{2}=\frac{k}{N(t)-1}+2 k^{\prime}\left(1-\frac{1}{N(t)-1}\right) .
$$

If we pull the end nodes of the network with force $F$ we find that there are $2^{l}$ shortcuts of the extension $\Delta_{x}=F /\left(K(t) 2^{l}\right)$. Here $l \in[0, t-2]$ is an integer that labels the shortcuts according to the step they have been added to the network. This in turn results in the following extension distribution

$$
P\left(\Delta_{x}\right) \sim\left(\Delta_{x}\right)^{-2}
$$

Now we start to increase $F$ from zero. In very small $F, R_{0}=1$. When the force reaches to $F_{1}=K(t) \delta_{c}$, the spring connecting node 1 to $N(t)$ extends by $\delta_{c}$ and tears. After this event, $R_{1}=2$ and the number of shortcuts decreases to $M_{1}=M(t)-1$. Moreover the effective network stiffness is given by $K_{1}=K(t-1) / 2$. It is easy to see that a shortcut connecting two far nodes has a larger extension than one connecting two near nodes. Thus 
we can summarize the behavior of interesting quantities versus $a$

$$
\begin{array}{r}
F_{a}=K(t-a+1), \\
R_{a}=2^{a}, \\
M_{a}=M(t)+1-2^{a}, \\
K_{a}=\frac{K(t-a)}{2^{a}} .
\end{array}
$$

The physical end-to-end distance then reads

$$
X_{a}=\frac{F_{a}}{K_{a}}=R_{a} \frac{K(t-a+1)}{K(t-a)}
$$

which for $t-a \gg 1$ is approximately equal to $R_{a}$. Thus in this limit the physical end-toend distance is well given by CEED. From the above relations we obtain $F_{a}=K_{a-1} R_{a-1}$. Consider the bonds of the shortest path connecting node 1 to $N . F_{a}$ is the force at which the extensions of these bonds become $\delta_{c}$. Note that, however, the relation between force and CEED is linear

$$
F_{a}=\frac{k R_{a}}{2^{t}}+2 k^{\prime}\left(1-\frac{R_{a}}{2^{t}}\right) .
$$

From Eqs. (3) one easily finds that

$$
\Delta R_{a}=-\Delta M_{a}=2^{a}, \quad \Delta F_{a}=\frac{k}{2^{t-a}}\left(1-\frac{2 k^{\prime}}{k}\right) .
$$

We see that for $k^{\prime}<k / 2, F_{a}$ increases versus $a$. Thus in this situation we have a continues change in $R$. In the continuum approximation we obtain

$$
P(\Delta R) \sim(\Delta R)^{-1}
$$

On the other hand, when $k^{\prime}>k / 2$, from Eqs. (3) and (16) we have $F_{1}=K(t)$ and $F_{1}>F_{2}>$ $F_{3} \ldots$. It means that after tearing the first shortcut, we reach a new configuration in which the extension of most extended shortcut(s) is(are) greater than $\delta_{c}$. This process continues until all shortcuts tear. Note that during this event which occurs at $F=F_{1}$, CEED changes from 1 to $N-1$. Thus we have an abrupt change of CEED at $F_{c}=K(t)$.

\section{COMPLETE NETWORKS}

In a complete network, each node is connected to all the other nodes. Thus the number of shortcuts is $M_{0}=(N-1)(N-2) / 2$. We start with the simple case of $k^{\prime}=k$ with the 


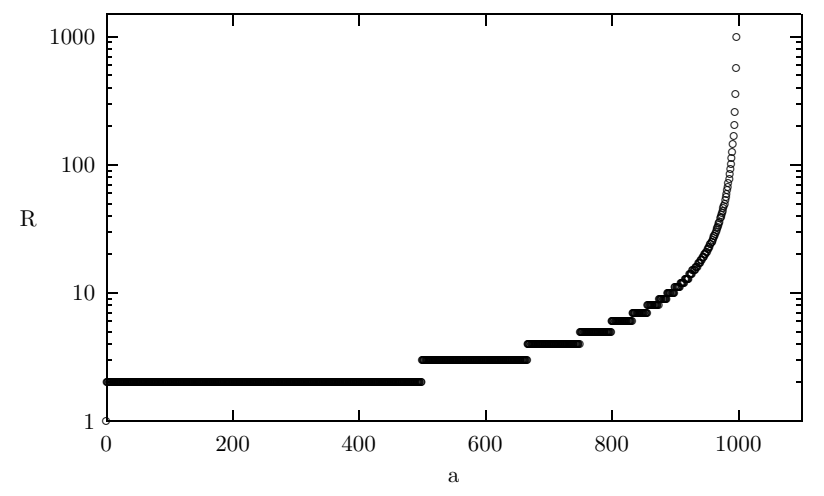

FIG. 2: Chemical end-to-end distance vs $a$ for a complete network of size $N=1000$ with $k=1$ and $k^{\prime}=0$.

effective network stiffness of $K_{0}=k N / 2$. Moreover, $P\left(\Delta_{x}\right)$ consists of three delta peaks corresponding to three different kinds of shortcuts in the network: The first kind is a single shortcut between the end nodes and has the largest extension. The second kind of shortcuts which have less extensions, are those that connect the end nodes to the inner nodes. And the remaining shortcuts, with zero extensions, are the shortcuts connecting the inner nodes to each other.

In the absence of external force, $R_{0}=1$. For a given $F$ we have the largest extension in the bond connecting node 1 to $N$. Hence by increasing $F$ the first avalanche occurs at $F_{1}=$ $k N / 2$. At this step we have $M_{1}=M_{0}-1, R_{1}=2$ and $K_{1}=k(N-2) / 2$. A simple calculation shows that for all springs connected to nodes 1 and $N$, we have $\Delta_{x}=F /(k(N-2))$. So by increasing $F$ we reach $F_{2}=k(N-2)$ in which $2(N-3)$ shortcuts are teared. In this step, we will have a complete network consisted of all nodes between 2 and $N-1$. But we know already that the spring connecting node 2 to $N-1$ can only stand forces lower than $k(N-2) / 2$ which is much smaller than $F_{2}$. Thus, in the second avalanche, all shortcuts will be removed. So $F_{c}=k(N-2)$ is the threshold $F$ in which CEED has an abrupt change.

Next we consider the simple case of $k^{\prime}=0$. It is clear that in this situation, the shortcuts have no contribution in the elastic properties of the network and we have a trivial problem in this respect. But in the process of increasing $F$, the effect of shortcuts in quantities like CEED is still important and nontrivial. It is not difficult to show that in this limit $P\left(\Delta_{x}\right) \propto\left(F N / k-\Delta_{x}\right)$. Moreover, as a function of the number of avalanches $a$, we have

$$
F_{a}=\frac{k}{N-a}
$$




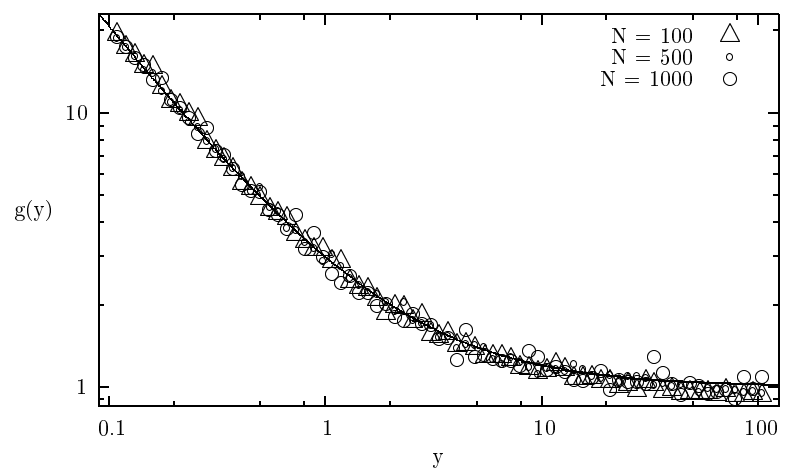

FIG. 3: $g(y)=2 K /(k p N)$ vs $y=p N^{2}$ for networks of size $N=100,500$ (averaged over 1000 realizations) and $N=1000$ (averaged over 300 realizations). The line represents the curve $1+2 / y$.

$$
\begin{array}{r}
R_{a}=1+\left[\frac{N}{N-a}\right]_{+}, \\
M_{a}=M_{0}-\frac{a(a+1)}{2}, \\
K_{a}=\frac{k}{N-1},
\end{array}
$$

where $[x]_{+}$denotes the smallest integer larger than or equal to $x$. In Fig. 2 we have shown $R$ versus $a$. Here again the relation between force and CEED is linear. If we take $a$ and $R_{a}$ as continuous variables and use Eq. (8) we obtain

$$
P(\Delta R) \sim(\Delta R)^{-3 / 2}
$$

This behavior is also seen in mean field models of fiber bundles for the size distribution of avalanches [18].

For $0<k^{\prime}<k, P\left(\Delta_{x}\right)$ still consists of three delta peaks. Numerical simulations show that only for $k^{\prime} \ll k$ the abrupt change of CEED is replaced by a continuous one (more precisely a staircaselike behavior). For example, if we choose $N=100$ and $k=1$ we obtain a nearly continuous transition only for $k^{\prime}<0.01$. We found that this value of $k^{\prime}$ is a decreasing function of network size. Thus for $N \rightarrow \infty$ a continues transition occurs only at $k^{\prime}=0$.

\section{RANDOM SMALL-WORLD NETWORKS}

We take a one-dimensional chain of $N$ nodes with $N-1$ regular bonds. Then with probability $p$, we connect any two nodes of distance larger than 1 by a shortcut. In the 


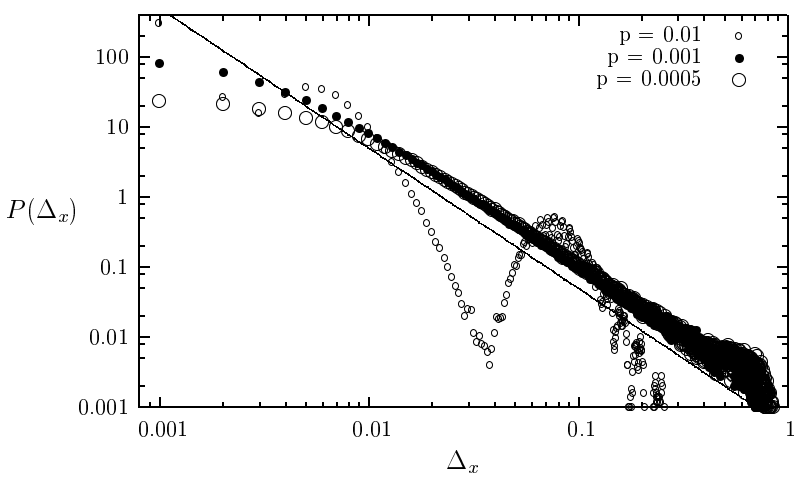

FIG. 4: Extension distribution for RSWNs of size $N=1000$ and $k=k^{\prime}=1$ averaged over 5000 realizations. The line displays a power law of exponent -2 .

following, we consider three different cases (i) $k=k^{\prime}$, (ii) $k^{\prime}=0$ and (iii) $0<k^{\prime}<k$.

\section{A. The case $k=k^{\prime}$}

For $p>1 / N$ we expect to have $K=k p N / 2$ which in the limit of $p=1$ is equal to the stiffness of a complete network. In other word if $p>1 / N$, we have effectively a complete network in which $k$ has been replaced with $k p$. On the other hand for $p=0$ and large $N$ we have $K=k / N$. Using these limiting cases we suggest that

$$
\frac{K}{N}=\frac{k p}{2} g\left(p N^{2}\right), \quad g(y) \sim\left\{\begin{array}{l}
1, y \gg 1 \\
\frac{2}{y}, y \rightarrow 0
\end{array}\right.
$$

Note that for large $N, p N^{2} / 2$ is equal to the average number of shortcuts. Numerical simulations shown in Fig. 3] support this scaling relation.

As $p$ decreases, we observe a crossover in $P\left(\Delta_{x}\right)$ from a three-modal behavior to a scale free one, see Fig. 4. When $p>1 / N$ this distribution has three broad maximums instead of three delta peaks in a complete network. The broadening of these peaks is due to the random structure of the SWN. Just in the small-world regime, $P\left(\Delta_{x}\right)$ is given by a power law distribution of power -2 . In this respect the RSWN belongs to the universality class of the hierarchical model introduced in section [II.

In our quasistatic process of increasing $F$, CEED changes nearly abruptly at $F_{c}$. This behavior has been shown in Fig. [5 that displays $P(\Delta R)$ for single realizations of the process. We observe that after a few events of small size, we have a large change of order $N$ in $R$ which 


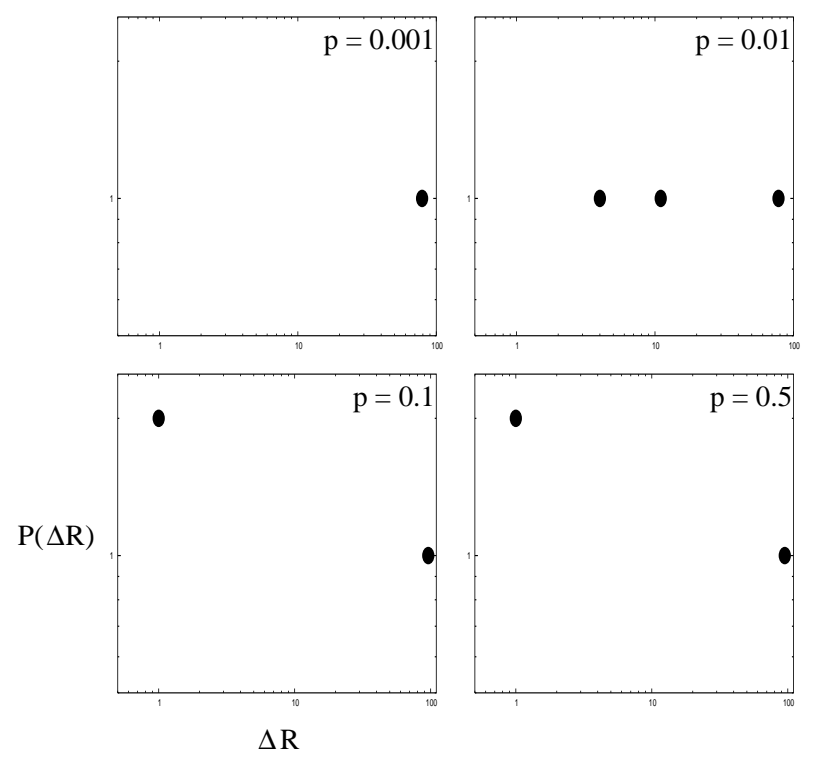

FIG. 5: $P(\Delta R)$ for single realizations with different $p$ when $k=k^{\prime}=1, N=100$. There are a few small changes in $R$ followed by a large change of order $N$ happened at critical force $F_{c}$.

we interpret it as a discontinuous behavior of CEED. Again for $p \gg 1 / N, F_{c}=k p(N-2)$, see Fig. 6. For $p \rightarrow 0$, when there is at least one shortcut in the network, $F_{c} \simeq k \delta_{c}$. These limiting behaviors of $F_{c}$ with $p$ have been shown in Fig. 6. We find that average number of shortcuts emanating from a node, $p N$, determines $F_{c}$. As far as the shortcuts do not overlap with each other, $F_{c}$ dose not depend on their number. Actually for a finite number of shortcuts, a typical configuration has almost nonoverlapping shortcuts. In this situation we have $F_{c} \simeq k \delta_{c}$. Thus we expect that $F_{c}$ changes considerably only when $p N \sim 1$. Moreover, from the linear feature of the system we also expect that $F_{c}$ should be proportional to $\delta_{c}$ and $k$. Thus, we suggest $F_{c}=k \delta_{c} h(p N)$, where $h(p N)$ is a dimensionless scaling function. In Fig. [6 we show that this relation works well for two small-size networks and within our numerical errors.

\section{B. The case $k^{\prime}=0$}

When $k^{\prime}=0$ the shortcuts have no contribution in the elastic properties of the network. As indicated in the study of complete networks, $P\left(\Delta_{x}\right)$ is a linear decreasing function. But notice that during the quasistatic process, tearing of shortcuts leads to considerable varia- 


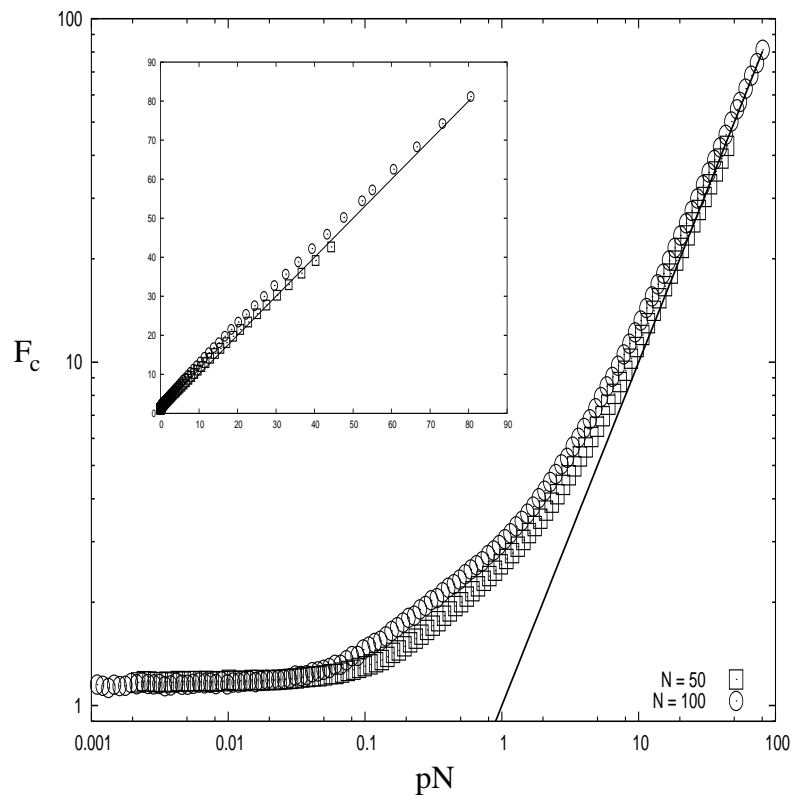

FIG. 6: $\quad F_{c}$ vs $p N$ in RSWNs with $k=k^{\prime}=1$ for $N=50$ and $N=100$ averaged over 5000 and 1000 realizations respectively. The solid line displays $F_{c}=k p N$. In each step of the quasistatic process we increase $F$ by 0.1 . The inset shows more clearly the linear behavior of $F_{c}$ for large $p N$.

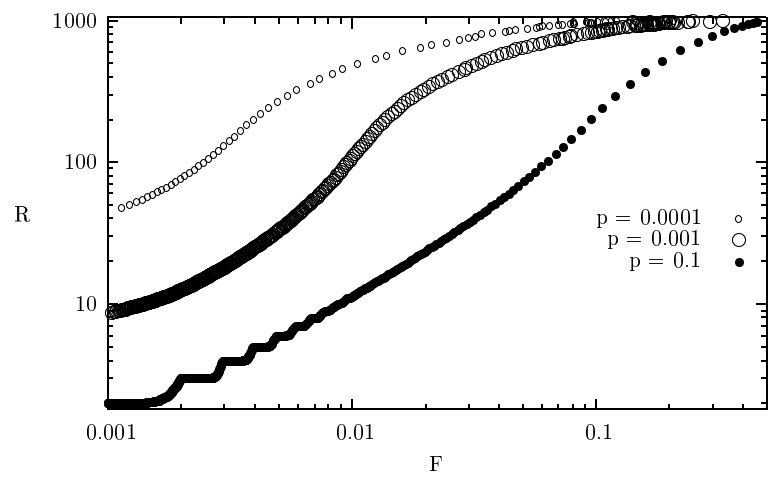

FIG. 7: The CEED vs $F$ in RSWNs of size $N=1000, k^{\prime}=0$ and $k=1$ after 5000 (for $p=$ $0.1,0.001$ ) and 10000 (for $p=0.0001$ ) realizations.

tions in $R$. In Fig. [7] we show variation of $R$ versus $F$ obtained by numerical simulations. There is no linear relation between $R$ and $F$. Indeed for a given $F$, all shortcuts connecting two nodes of distance larger than $L_{F}=k / F$ have been already teared. This introduces another length scale. We expect $R$ to be a function of the number of shortcuts in a subnetwork of size $L_{F}$, that is,

$$
\frac{R}{N}=f\left(p L_{F}^{2}\right)
$$




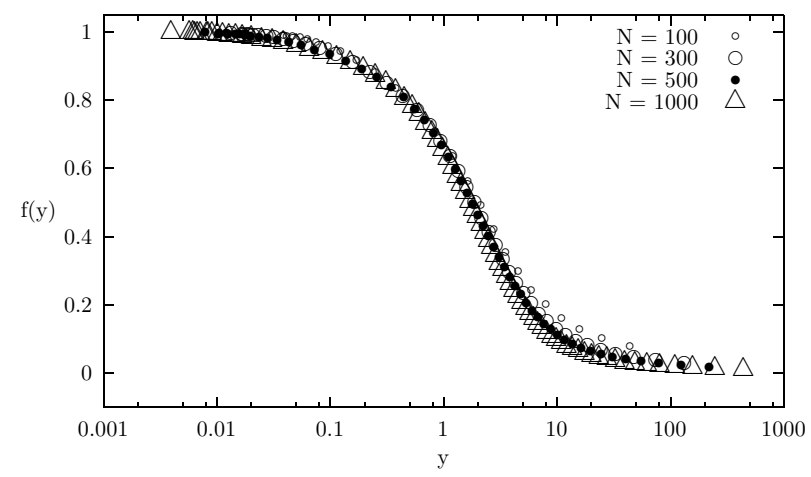

FIG. 8: $f(y)=R / N$ vs $y=p L_{F}^{2}$ for RSWNs of different sizes (averaged over 10000 realizations).

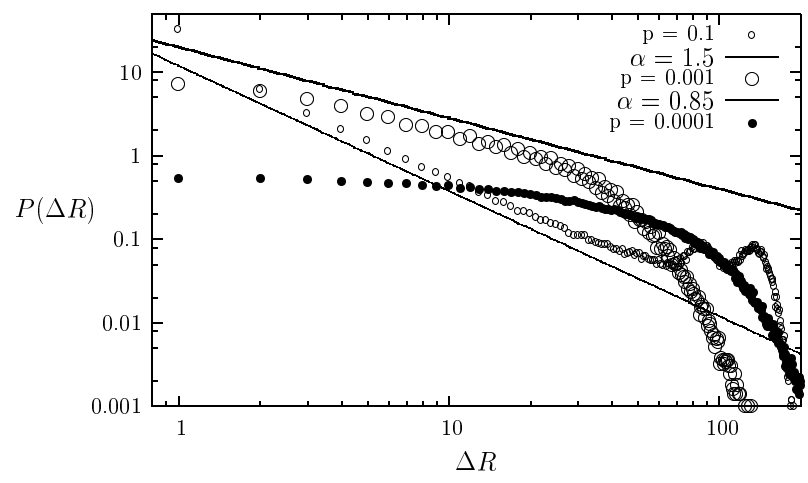

FIG. 9: $P(\Delta R)$ for RSWNs of size $N=1000, k^{\prime}=0$ and $k=1$. The lines display $P(\Delta R) \propto$ $(\Delta R)^{-\alpha}$. Number of realizations are the same as those of Fig. [6]

Numerical simulations presented in Fig. 8 confirm this scaling relation.

Figure 9 displays $P(\Delta R)$ for various values of $p$. For a small value of $p=0.1, P(\Delta R) \propto$ $(\Delta R)^{-3 / 2}$ like a complete network. For smaller $p$, we find a power law distribution of lower exponent. For a very small $p, P(\Delta R)$ is nearly a constant function with an exponential tail.

\section{The case $0<k^{\prime}<k$}

As before we take $k=1$ and decrease $k^{\prime}$ from one. Numerical simulations show that in this case the network stiffness is proportional to $k^{\prime}$. The effects of $k^{\prime}$ and $p$ on elastic properties are the same. It means that with respect to elastic properties, the decreasing of $k^{\prime}$ at a fixed $p$ is equivalent to the decreasing of $p$ at a fixed $k^{\prime}$. In Fig. 10 we display the extension distribution when $k^{\prime}=0.1$. It is observed that when $p>1 / N$ we have a 


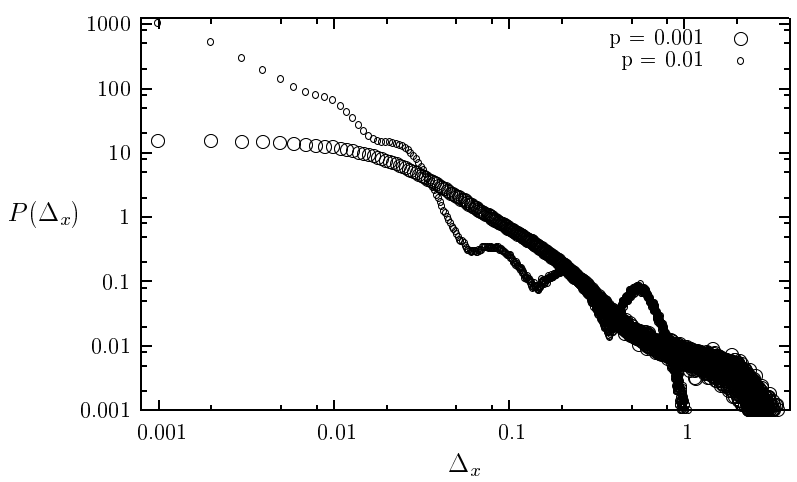

FIG. 10: Extension distribution for RSWNs of size $N=1000, k=1$ and $k^{\prime}=0.1$ averaged over 5000 realizations.

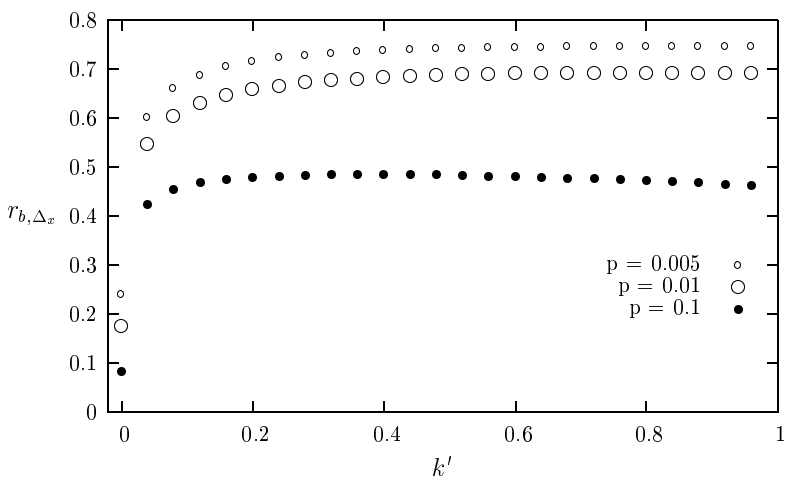

FIG. 11: Correlation coefficient of $\Delta x$ and $b$ for $N=100$ after averaging over 5000 realizations.

multimodal distribution in contrast to the three-modal distribution of case $k=k^{\prime}$ for the same value of $p$. Recall that we did not have such a behavior for lower values of $p$ when $k=k^{\prime}$. Indeed this new multimodal behavior is observed only when we have a large number of shortcuts.

The extension distribution of RSWNs shows that there are some shortcuts bearing very large forces compared with the other ones. Certainly this is because of their essential roles in the network structure. A good measure of centrality of bonds in a network is their betweenness 2]. Suppose that we have $n$ shortest pathes connecting node 1 to $N$. A given shortcut may contribute in $n_{s}$ of these pathes. Then, the betweenness of this shortcut is defined as $b=n_{s} / n$. We define

$$
r_{b, \Delta_{x}}:=\frac{<b \Delta_{x}>-<b><\Delta_{x}>}{\sqrt{\sigma_{b} \sigma_{\Delta_{x}}}},
$$

as a measure of correlation between extension and betweenness of shortcuts. In this definition $\sigma_{\Delta_{x}}$ and $\sigma_{b}$ are variances of $\Delta_{x}$ and $b$, respectively. In Fig 11 we show how the 


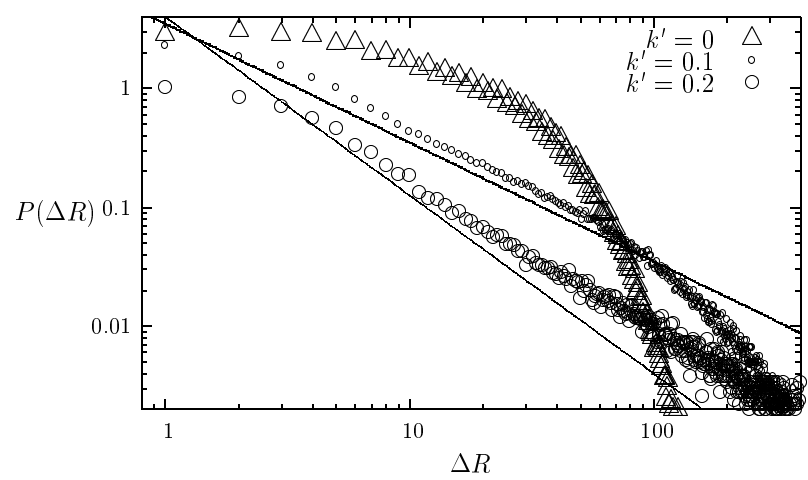

FIG. 12: $P(\Delta R)$ for a network with $N=1000, p=0.001$ and $k=1$. The data are results of averaging over $10000\left(k^{\prime}=0\right)$ and $5000\left(k^{\prime}=0.1,0.2\right)$ realizations. The lines represent power laws of exponent -1 and $-3 / 2$.

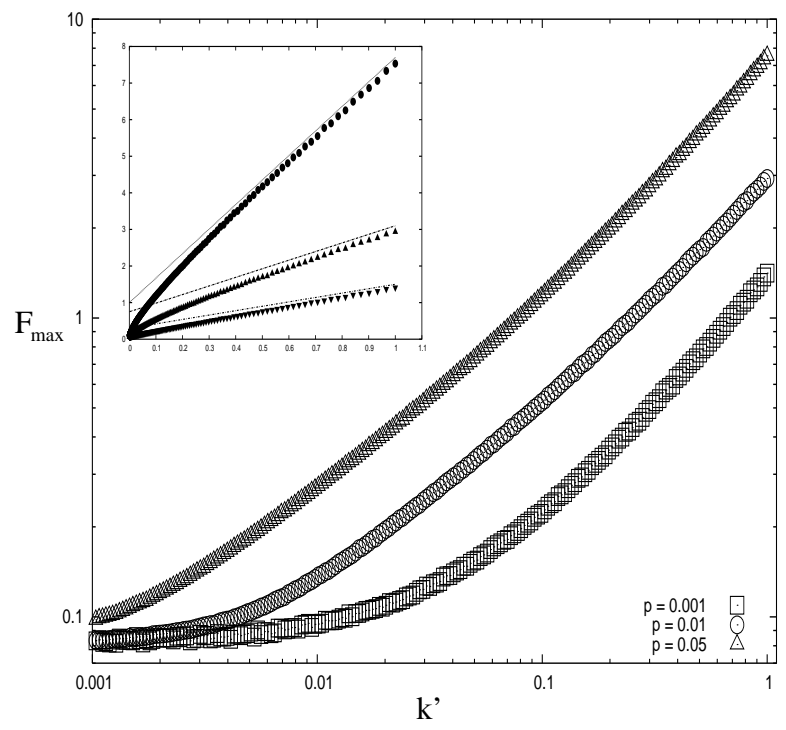

FIG. 13: $F_{\max }$ vs $k^{\prime}$ for $N=100$ and $k=1$ (averaged over 1000 realizations). The inset shows more clearly the linear behavior of data for large $k^{\prime}$. The lines display linear functions $F_{\max }=a_{0}+a_{1} k^{\prime}$.

correlation coefficient depends on $k^{\prime}$ and $p$. As expected, $r_{b, \Delta_{x}}$ has a considerable positive value in the small-world regime. The correlation coefficient is nearly independent of $k^{\prime}$ except for a rapid decrease to zero for $k^{\prime} \rightarrow 0$.

In the quasistatic process, as it happens in complete networks, a considerable number of avalanches takes place only for a significantly small $k^{\prime}$. Indeed, the larger $p$ the lower value of $k^{\prime}$; we need to see a continuous behavior of $R$. In Fig. 12 we compare $P(\Delta R)$ for two cases of $k^{\prime}=0$ and $k^{\prime}=0.1$. The figure shows that as expected, by increasing $k^{\prime}, P(\Delta R)$ ap- 
proaches to that of a complete network. Indeed, in the small-world regime and for a nonzero $k^{\prime}, P(\Delta R)$ obeys a power law of exponent $-\alpha$ with $\alpha<3 / 2$. This exponent approaches to $-3 / 2$ as we increase $k^{\prime}$ or $p$.

In this case it is not easy to define $k_{c}^{\prime}(p)$, the value which separates continuous and discontinuous regimes. Instead we calculate $F_{\max }$, the force which leads to $R_{\max }=N-1$. We

expect that a change in the behavior of $F_{\max }$ with respect to $k^{\prime}$ signals a crossover from a continuous to a discontinuous region. In Fig. [13] we show the variation of $F_{\text {max }}$ versus $k^{\prime}$ obtained by numerical simulation. We see that by decreasing $k^{\prime}$, the linear behavior of $F_{\text {max }}$ changes and then it saturates for $k^{\prime} \rightarrow 0$. This crossover occurs for a lower $k^{\prime}$ as one enhances the number of shortcuts.

\section{CONCLUSIONS}

We studied the static and quasistatic properties of SWNs. We observed that the network's structure significantly affects the static and quasistatic behavior. Thus, we could draw some conclusions about the structure of networks through the study of these properties.

The summary of the main results for RSWNs have been represented in table \ and Fig. 14. We found that for very small values of $p$, the effective stiffness of the network is comparable with that of a complete network. When $k=k^{\prime}$ the extension distribution of shortcuts is a power law of exponent -2. In this respect RSWNs behave like a hierarchical network introduced in this paper. There was also a strong positive correlation between the betweenness of a shortcut and its extension. It means that just by looking at the distribution of extensions in a network, one could be able to distinguish which shortcuts are more central.

In the quasistatic part, we showed that by increasing $F$, CEED could have a continuous or discontinuous transition. In general, to have a continuous transition we need a much smaller spring constant for shortcuts rather than regular bonds. In the case of a discontinuous transition, the critical force is determined by the average number of shortcuts per node. It was found that for a continuous transition, $P(\Delta R)$ is given by power law distributions of the exponent $-\alpha$ with $\alpha \leq 3 / 2$. 


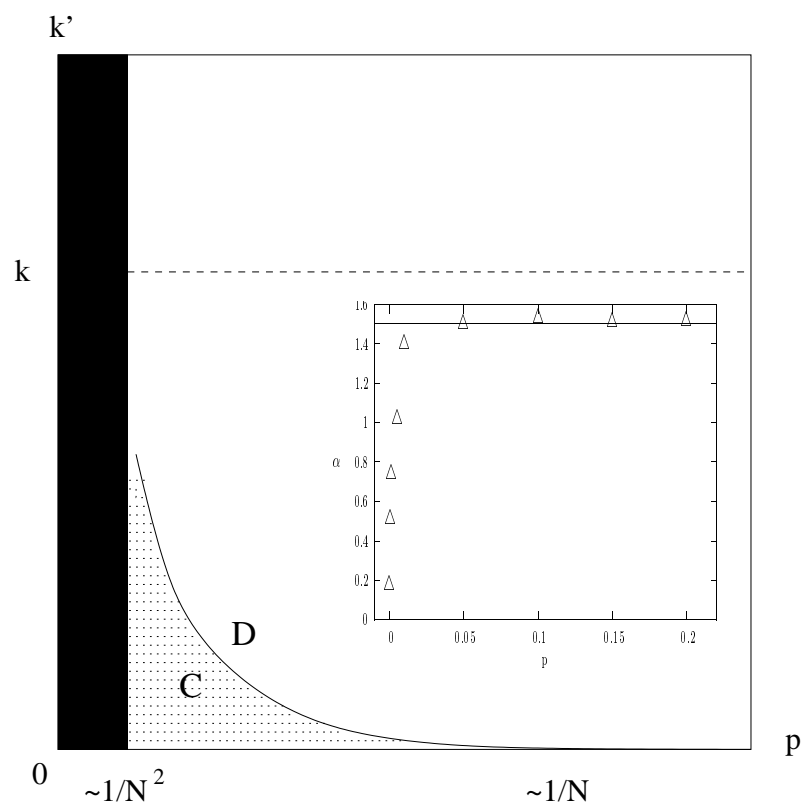

FIG. 14: Schematic representation of phase diagram. Dashed region indicates where we have a continuous behavior of $R$ vs. $F$. For $p<1 / N^{2}$ (black region) we can not speak of continuous or discontinuous behavior of $R$. Note that as $N$ approaches to infinity the continuous region reduces to the line $k^{\prime}=0$. The inset shows the variation of $\alpha$ with $p$ when $N=1000, k=1$ and $k^{\prime}=0$. The errors in $\alpha$ are of order 0.01. The same behavior will be observed for $\alpha$ when we fix $p$ and increase $k^{\prime}$. The solid line in inset indicates the expected value of $\alpha$ for large $p$ i.e. $3 / 2$.

\begin{tabular}{|c|c|c|}
\hline & $P\left(\Delta_{x}\right)$ & $P(\Delta R)$ \\
\hline$k=k^{\prime}$ & $\left(\Delta_{x}\right)^{-2}$ & $\delta_{\Delta R, N}($ an abrupt change of R) \\
\hline$k^{\prime}=0$ & $a_{0}-a_{1} \Delta_{x}$ & $(\Delta R)^{-\alpha}(\alpha \leq 3 / 2)$ \\
\hline $0<k^{\prime}<k$ & $\left(\Delta_{x}\right)^{-\beta}(\beta \leq 2)+$ oscillations (for large $\left.p\right)$ & $\left\{\begin{array}{l}(\Delta R)^{-\alpha}(\alpha \leq 3 / 2), \\
k^{\prime}<k_{c}^{\prime}(p) \\
\delta_{\Delta R, N},\end{array}\right.$ \\
\hline
\end{tabular}

TABLE I: Summary of results for $P\left(\Delta_{x}\right)$ and $P(\Delta R)$ in different cases. Here $k_{c}^{\prime}(p)$ gives the line in $\left(p, k^{\prime}\right)$ plane that separates continuous and discontinues behavior of CEED.

\section{Acknowledgments}

We would like to thank S. N. Rasuli for useful discussions. We are grateful to MirFaez Miri for comments and careful reading of the manuscript. 
[1] R. Albert and A. -L. Barabási, Rev. Mod. Phys. 74, 47 (2002).

[2] M. E. J. Newman, SIAM Review 45, 167 (2003).

[3] D. J. Watts and S. H. Strogatz, Nature 393, 440 (1998).

[4] L. A. N. Amaral, A. Scala, M. Barthélémy, and H. E. Stanly, Proc. Natl. Acad. Sci USA 97, $11149(2000)$.

[5] A. Barrat, M. Weigt, Europ. Phys. J. B 13, 547 (2000).

[6] L. de Arcangelis, H.J. Herrmann, Physica A 308(1-4), 545 (2002).

[7] A. D. Sanchez, J. M. Lopez, M. A. Rodriguez, Phys. Rev. Lett. 88, 048701 (2002).

[8] C. Castellano, D. Vilone, A. Vespignani, Europhys. Lett. 63, 153 (2003).

[9] E. Almaas, R. V. Kulkarni, D.Stroud, Phys. Rev. E 68, 056105 (2003).

[10] K. Medvedyeva, P. Holme, P. Minnhagen, B. J. Kim, Phys. Rev. E 67, 036118 (2003).

[11] L. G. Morelli, G. Abramson, M. N. Kuperman, Eur. Phys. J. B 38, 495 (2004).

[12] S. Jespersen, I. M. Sokolov and A. Blumen, Phys. Rev. E 62, 4405 (2000).

[13] S. Jespersen, I. M. Sokolov, A. Blumen, Arxiv preprint cond-mat/0004392

[14] S. Jespersen, A. Blumen, Phys. Rev. E, 62, 6270 (2000) .

[15] M. Vendruscolo, N. V. Dokholyan, E. Paci, M. Karplus, Phys. Rev. E 65, 061910 (2002).

[16] Y. Kantor, M. Kardar, Phys. Rev. E 54, 5263 (1996).

[17] P. Sen, B. K. Chakrabarti, J. Phys. A: Math. Gen, 34 (38), 7749 (2001).

[18] D. Sornette, J. Phys. A 22, L243 (1989).

[19] P.C. Hemmer and A. Hansen, J. Appl. Mech. 59, 909 (1992). 